\title{
Some Characterizations of Curves in n-dimensional Euclidean Space $\mathbf{I E}^{\mathbf{n}}$
}

\section{Sezgin BÜYÜKKÜTÜK ${ }^{1 *}$, İlim Kİșí ${ }^{2}$ Günay ÖZTÜRK ${ }^{3}$, Kadri ARSLAN $^{4}$}

\begin{abstract}
In this work, we deal with a curve whose position vector can be expressed with the help of Frenet Frame in $n$-dimensional Euclidean space $I E^{n}$. We classify this type of curve with regards to curvature functions and get certain consequences for $T$-constant, $N$-constant and constant ratio curves in $I E^{n}$.
\end{abstract}

Keywords: Constant ratio curves, $W$-curves, position vector

\footnotetext{
1 Sezgin BÜYÜKKÜTÜK (Orcid ID: 0000-0002-1845-0822), Kocaeli Üniversitesi, Gölcük Meslek Yüksekokulu, Kocaeli, Türkiye

2 İlim KİŞİ (Orcid ID: 0000-0002-4785-8165), Kocaeli Üniversitesi, Fen Edebiyat Fakültesi, Matematik Bölümü, Kocaeli, Türkiye

${ }^{3}$ Günay ÖZTÜRK (Orcid ID: 0000-0002-1608-0354), İzmir Demokrasi Üniversitesi, Fen Edebiyat Fakültesi, Matematik Bölümü, İzmir, Türkiye

${ }^{4}$ Kadri ARSLAN (Orcid ID: 0000-0002-1440-7050), Uludağ Üniversitesi, Fen Edebiyat Fakültesi, Matematik Bölümü, Bursa, Türkiye

*Sorumlu Yazar/Corresponding Author: Sezgin BÜYÜKKÜTÜK, e-mail: sezgin.buyukkutuk@kocaeli.edu.tr
} 


\section{INTRODUCTION}

A unit speed curve is described by most geometers, as a curve $x=x(s)$ whose parametrization is written by arclength parameter s. So as to define curvatures of a curve, the well-known Frenet formulas can be explained as follows:

A regular curve $x=x(s): I \subset I R \rightarrow I E^{3}$, given with the arclength parameter, has at least four continuous derivatives. Denote by the tangent vector $T=x^{\prime}(s)$; we suppose $T^{\prime}(s)$ is nonzero for all s. Then, it is possible to define the principle normal vector field $N_{1}$ and the curvature $\kappa_{1}$ by $T^{\prime}(s)=\kappa_{1} N_{1}$. Since the vectors $T$ and $N_{1}$ are orthogonal, the binormal vector field is yield by the relation $N_{2}^{\prime}=-\kappa_{2} N_{1}$ defines the torsion $\kappa_{2} \cdot \kappa_{1}$ and $\kappa_{2}$ are also called the first and the second curvatures of the curve.

The Frenet formulas are

$$
\begin{aligned}
& T^{\prime}=\kappa_{1} N_{1}, \\
& N_{1}^{\prime}=-\kappa_{1} T+\kappa_{2} N_{2}, \\
& N_{2}^{\prime}=-\kappa_{2} N_{1} .
\end{aligned}
$$

The significant planes in $I E^{3}$ are osculating, rectifying and normal planes which are spanned by $\left\{T, N_{1}\right\},\left\{T, N_{2}\right\}$, and $\left\{N_{1}, N_{2}\right\}$, respectively. If a curve's position vector is orthogonal to its normal vector field, then it is called a rectifying curve in $I E^{3}$. In $n$-dimensional Euclidean space, we have a similar definition as 3-dimensional Euclidean space. In $I E^{n}$, rectifying curve's position vector is spanned by $\left\{T, N_{2}, N_{3}, \ldots, N_{n-1}\right\}$.

B.Y. Chen introduces the notion of the rectifying curves in his study(Chen, 2003). In that paper, he gives a basic classification of rectifying curves. Especially, in (Chen et al., 2005), the author gives the connection between these curves and centrodes that have a place in kinematics and mechanics. Also in the study (Illarslan et al., 2008), the authors give some characterizations about rectifying curves. Recently in (Cambie et al., 2016), rectifying curves have been studied in arbitrary Euclidean space.

In (Chen, 2001), B.Y. Chen gives a new concept for Euclidean spaces: constant ratio curves. The author presents in this study which curves satisfy the condition of being a constant ratio curve. Also in (Chen, 2002), B.Y. Chen announces the notion of $N$-constant and $T$-constant types of curves. In studies (Büyükkütük et al., 2015, 2016, 2017; Gürpınar et al., 2015; Kişi et al., 2015, 2017, 2018, Öztürk et al., 2017, 2018), by using Frenet and Bishop frame, the necessary and sufficient conditions are given for curves to become $T$-constant and $N$-constant, respectively.

In this study, we deal with any curve in $n$-dimensional Euclidean space $I E^{n}$ with regard to its Frenet frame $\left\{T, N_{1}, N_{2}, \ldots, N_{n-1}\right\}$. So, we can write the parametrization of the curve as:

$$
x(s)=m_{0}(s) T(s)+\sum_{i=1}^{n-1} m_{i}(s) N_{i}(s)
$$


where the functions $m_{i}(s), i=0,1,2, . ., n-1$ are differentiable. We classify this curve with regards to curvature functions and obtain the sufficient and necessary conditions for this curve to become $T-$ constant, $N$-constant and constant-ratio.

\section{BASIC NOTATION}

In $n$-dimensional Euclidean space, a unit speed curve $x: I \rightarrow I E^{n}$ can be described by the Frenet frame, which is associated with the vectors $T, N_{1}, N_{2}, \ldots, N_{n-1}$ and n-1 functions $\kappa_{1}, \kappa_{2}, \ldots, \kappa_{n-1}: I \rightarrow I R$. Thus, for $n$-dimensional Euclidean space, Frenet formulas can be given by

$$
\left[\begin{array}{c}
T^{\prime} \\
N_{1}^{\prime} \\
N_{2}^{\prime} \\
\cdot \\
\cdot \\
\cdot \\
N_{n-2}^{\prime} \\
N_{n-1}^{\prime}
\end{array}\right]=\left[\begin{array}{cccccccc}
0 & \kappa_{1} & 0 . & . & . & . & 0 & 0 \\
-\kappa_{1} & 0 & \kappa_{2} & . & . & . & 0 & 0 \\
0 & -\kappa_{2} & 0 & . & . & . & 0 & 0 \\
. & . & . & . & . & . & . & . \\
. & . & . & . & . & . & . & . \\
. & . & . & . & . & . & . & . \\
0 & 0 & . & . & . & . & . & \kappa_{n-1} \\
0 & 0 & . & . & . & . & -\kappa_{n-1} & 0
\end{array}\right]\left[\begin{array}{c}
T \\
N_{1} \\
N_{2} \\
\cdot \\
. \\
. \\
N_{n-2} \\
N_{n-1}
\end{array}\right] .
$$

If any curve has constant Frenet curvatures, then it is known as $W$-curve. A parametrization of a unit speed $W$-curve in $I E^{2 r}$ is represented by

$$
x(s)=a_{0}+\sum_{i=1}^{r}\left(a_{i} \cos \mu_{i} s+b_{i} \sin \mu_{i} s\right)
$$

and a unit speed $W$-curve in $I E^{2 r+1}$ is represented by

$$
x(s)=a_{0}+b_{0} s+\sum_{i=1}^{r}\left(a_{i} \cos \mu_{i} s+b_{i} \sin \mu_{i} s\right)
$$

where $a_{0}, b_{0}, a_{1}, \ldots, a_{r}, b_{1}, \ldots, b_{r}$ are constant vectors in $I E^{n}$ and $\mu_{1}<\mu_{2}<\ldots<\mu_{r}$ are real numbers( Gluck, 1966; Gray, 1993; Klein et al., 1871).

\section{CHARACTERIZATIONS OF CURVES IN IE ${ }^{\text {n }}$}

Now, we consider curves given with Equality 2. Differentiating Eq. 2. with regard to arclength parameter, we get

$$
x^{\prime}(s)=m_{0}^{\prime}(s) T(s)+m_{0}(s) T^{\prime}(s)+\sum_{i=1}^{n-1}\left(m_{i}^{\prime}(s) N_{i}(s)+m_{i}(s) N_{i}^{\prime}(s)\right) .
$$

Then, by the use of Frenet formulas 3., we yield

$$
\begin{aligned}
T(s)= & m_{0}^{\prime}(s) T(s)+m_{0}\left(\kappa_{1}(s) N_{1}(s)\right) \\
& +m_{1}^{\prime}(s) N_{1}(s)+m_{1}(s)\left(-\kappa_{1}(s) T(s)+\kappa_{2}(s) N_{2}(s)\right)+\sum_{i=2}^{n-1}\left(m_{i}^{\prime}(s) N_{i}(s)\right) \\
& +\sum_{i=2}^{n-2} m_{i}(s)\left(-\kappa_{i}(s) N_{i-1}(s)+\kappa_{i+1}(s) N_{i+1}(s)\right)-\kappa_{n-1}(s) N_{n-2}(s) .
\end{aligned}
$$

Equivalently, 


$$
\begin{aligned}
T(s)= & \left(m_{0}^{\prime}(s)-\kappa_{1}(s) m_{1}(s)\right) T(s) \\
& +\sum_{i=2}^{n-2}\left(m_{i}^{\prime}(s)+\kappa_{i}(s) m_{i-1}(s)-\kappa_{i+1}(s) m_{i+1}(s)\right) N_{i}(s) \\
& +\left(m_{n-1}^{\prime}(s)+\kappa_{n-1}(s) m_{n-2}(s)\right) N_{n-1}(s) .
\end{aligned}
$$

It follows that,

$m_{0}^{\prime}(s)-\kappa_{1}(s) m_{1}(s)=1$

$m_{1}^{\prime}(s)+\kappa_{1}(s) m_{0}(s)-\kappa_{2}(s) m_{2}(s)=0$,

$m_{i}^{\prime}(s)+\kappa_{i}(s) m_{i-1}(s)-\kappa_{i+1}(s) m_{i+1}(s)=0 \quad$ with $i \in\{2,3, \ldots, n-2\}$,

$m_{n-1}^{\prime}(s)+\kappa_{n-1}(s) m_{n-2}(s)=0$.

The functions $m_{i}(s), 1 \leq i \leq n-1$, can be given with the Frenet curvatures, the curvature function $\mathrm{m}_{0}(\mathrm{~s})$, and their derivatives. From the Equation system 9.,

$m_{1}(s)=\frac{m_{0}^{\prime}(s)-1}{\kappa_{1}(s)}$

and

$m_{2}(s)=\frac{\kappa_{1}^{2}(s) m_{0}(s)}{\kappa_{2}(s)\left(m_{0}^{\prime}(s)-1\right)}\left(\frac{m_{0}^{\prime}(s)-1}{\kappa_{1}(s)}\right)+\frac{1}{\kappa_{2}(s)}\left(\frac{m_{0}^{\prime}(s)-1}{\kappa_{1}(s)}\right)^{\prime}$.

Introducing functions $m_{1,0}, m_{2,0}$, and $m_{2,1}$, we rewrite these equations as

$$
\begin{aligned}
& m_{1}(s)=m_{1,0}(s) \frac{m_{0}^{\prime}(s)-1}{\kappa_{1}(s)}, \quad(12) \\
& m_{2}(s)=m_{2,0}(s)\left(\frac{m_{0}^{\prime}(s)-1}{\kappa_{1}(s)}\right)+m_{2,1}(s)\left(\frac{m_{0}^{\prime}(s)-1}{\kappa_{1}(s)}\right)^{\prime} .
\end{aligned}
$$

By induction method for the Equation system 9., we get

$$
m_{i}(s)=\sum_{k=0}^{i-1} m_{i, k}(s) \frac{\partial^{k}}{\partial s^{k}}\left(\frac{m_{0}^{\prime}(s)-1}{\kappa_{1}(s)}\right)
$$

for $i \in\{3,4, \ldots, n-1$,$\} . Inductively, we can give the functions m_{i, k}$ by the following system

$$
\left\{\begin{array}{l}
m_{1,0}=1 \\
m_{2,0}=\frac{\kappa_{1}^{2}(s) m_{0}(s)}{\kappa_{2}(s)\left(m_{0}^{\prime}(s)-1\right)}, \quad m_{2,1}=\frac{1}{\kappa_{2}(s)}
\end{array}\right.
$$

and for $i \in\{3,4, \ldots, n-1$,$\} ,$ 


$$
\left\{\begin{array}{l}
m_{i, 0}(s)=\frac{m_{i-1,0}^{\prime}(s)+\kappa_{i-1}(s) m_{i-2,0}(s)}{\kappa_{i}(s)} \\
m_{i, k}(s)=\frac{m_{i-1, k}^{\prime}(s)+m_{i-1, k-1}(s)+\kappa_{i-1}(s) m_{i-2, k}(s)}{\kappa_{i}(s)}, \text { for } k \in\{1,2, \ldots, i-3\} \\
m_{i, i-2}(s)=\frac{m_{i-1, i-2}^{\prime}(s)+m_{i-1, i-3}(s)}{\kappa_{i}(s)} \\
m_{i, i-1}(s)=\frac{m_{i-1, i-2}(s)}{\kappa_{i}(s)}
\end{array}\right.
$$

Theorem 1: Let a unit speed curve $x: I \subset I R \rightarrow I E^{n}$ has nonzero curvatures. Thus, we have

$$
\kappa_{n-1}(s) \sum_{k=0}^{n-3} m_{n-2, k}(s) \frac{\partial^{k}}{\partial s^{k}}\left(\frac{m_{0}^{\prime}(s)-1}{\kappa_{1}(s)}\right)+\left(\sum_{k=0}^{n-2} m_{n-1, k}(s) \frac{\partial^{k}}{\partial s^{k}}\left(\frac{m_{0}^{\prime}(s)-1}{\kappa_{1}(s)}\right)\right)^{\prime}=0,
$$

where $m_{i, k}$ are inductively defined by the Systems 15 . and 16 .

Theorem 2: Let a unit speed curve $x: I \subset I R \rightarrow I E^{n}$ has the parametrization 2 .

(i) If the curve is unit speed $W-$ curve in $I E^{n}(\mathrm{n}=2 \mathrm{r})$, then it satisfies the vectorial equation

$$
m_{0}^{(n)}+\sum_{k=1}^{\frac{n}{2}} a_{k} m_{0}^{(n-2 k)}=0
$$

(ii) If the curve is unit speed $W$-curve in $I E^{n}(\mathrm{n}=2 \mathrm{r}+1)$, then it satisfies the vectorial equation

$$
m_{0}^{(n)}+\sum_{k=1}^{\frac{n-1}{2}} a_{k} m_{0}^{(n-2 k)}-a_{\frac{n+1}{2}}=0,
$$

where

$$
\begin{aligned}
& a_{1}=\sum_{i=1}^{n-1} \kappa_{i}^{2}, \\
& a_{2}=\sum_{i=3}^{n-1} \kappa_{i}^{2}\left(\sum_{j=1}^{i-2} \kappa_{j}^{2}\right), \\
& a_{3}=\sum_{i=5}^{n-1} \kappa_{i}^{2}\left(\sum_{j=3}^{i-2} \kappa_{j}^{2}\left(\sum_{t=1}^{j-2} \kappa_{t}^{2}\right)\right),
\end{aligned}
$$




$$
\begin{aligned}
& a_{k}=\sum_{i=2 k-1}^{n-1} \kappa_{i}^{2}\left(\sum_{j=2 k-3}^{i-2} \kappa_{j}^{2}\left(\sum_{t=2 k-5}^{j-2} \kappa_{t}^{2}\left(\sum \ldots\right)\right)\right), \\
& a_{\frac{n-1}{2}}=\prod_{i=1}^{\frac{n-1}{2}} \kappa_{2 i-1}^{2}, \\
& a_{\frac{n}{2}}=\prod_{i=1}^{\frac{n}{2}} \kappa_{2 i-1}^{2}, \\
& a_{\frac{n+1}{2}}=\prod_{i=1}^{\frac{n+1}{2}} \kappa_{2 i}^{2},
\end{aligned}
$$

Proof. Let a unit speed curve $x: I \subset I R \rightarrow I E^{n}$ has the parametrization 2. By the use of the first equation in Eq. 9., we write

$$
m_{1}(s)=\frac{m_{0}^{\prime}(s)-1}{\kappa_{1}(s)}
$$

Since all curvatures are real constants, we get

$$
m_{1}^{\prime}=\frac{m_{0}^{\prime \prime}}{\kappa_{1}} .
$$

Putting this value into the second equation in Eq. 9., we write

$$
m_{2}^{\prime}=\frac{m_{0}^{\prime \prime}}{\kappa_{1} \kappa_{2}}+\frac{\kappa_{1}}{\kappa_{2}} m_{0} .
$$

In this way, by induction, for odd $\alpha$ and for even $\beta$, we obtain

$$
m_{\alpha}=\frac{m_{0}^{(\alpha)}}{\prod_{i=1}^{\alpha} \kappa_{i}}+\frac{\sum_{i=1}^{\alpha-1} \kappa_{i}^{2}}{\prod_{i=1}^{\alpha} \kappa_{i}} m_{0}^{(\alpha-2)}+\frac{\sum_{i=3}^{\alpha-1} \kappa_{i}^{2}\left(\sum_{j=1}^{i-2} \kappa_{j}^{2}\right)}{\prod_{i=1}^{\alpha} \kappa_{i}} m_{0}^{(\alpha-4)}+\ldots-\frac{\prod_{i=1}^{\frac{\alpha-1}{2}}\left(\kappa_{2 i}\right)^{2}}{\prod_{i=1}^{\alpha} \kappa_{i}}
$$

and

$$
m_{\beta}=\frac{m_{0}^{(\beta)}}{\prod_{i=1}^{\beta} \kappa_{i}}+\frac{\sum_{i=1}^{\beta-1} \kappa_{i}^{2}}{\prod_{i=1}^{\beta} \kappa_{i}} m_{0}^{(\beta-2)}+\frac{\sum_{i=3}^{\beta-1} \kappa_{i}^{2}\left(\sum_{j=1}^{i-2} \kappa_{j}^{2}\right)}{\prod_{i=1}^{\beta} \kappa_{i}} m_{0}^{(\beta-4)}+\ldots+\frac{\prod_{i=1}^{\frac{\beta}{2}}\left(\kappa_{2 i-1}\right)^{2}}{\prod_{i=1}^{\beta} \kappa_{i}} m_{0 .}
$$

We substitute these results into the last equation of the System 9., so obtain the differential equations 18. and 19., which completes the proof of the theorem.

Using Theorem 2, we can get all curvature functions. Hence, we have the following result:

Corollary 1: Let a unit speed curve $x: I \subset I R \rightarrow I E^{n}$ has the parametrization 2 .

(i) If the curve is unit speed $W$-curve in $I E^{n} \quad(\mathrm{n}=2 \mathrm{r})$, then the curvature functions of the curve are 
$m_{0}(s)=\sum_{i=1}^{\frac{n}{2}} c_{i} e^{-\lambda_{i} s}+\sum_{i=1}^{\frac{n}{2}} d_{i} e^{\lambda_{i} s}$,
$m_{1}(s)=\frac{1}{\kappa_{1}}\left(\sum_{i=1}^{\frac{n}{2}}-c_{i} \lambda_{i} e^{-\lambda_{i} s}+\sum_{i=1}^{\frac{n}{2}} d_{i} \lambda_{i} e^{\lambda_{i} s}-1\right)$,

$m_{j}(s)=\sum_{i=1}^{\frac{n}{2}} c_{i j} e^{-\lambda_{i} s}+\sum_{i=1}^{\frac{n}{2}} d_{i j} e^{\lambda_{i} s}, \quad j=2,4, \ldots, n-2$

$m_{k}(s)=\sum_{i=1}^{\frac{n}{2}} c_{i k} e^{-\lambda_{i} s}+\sum_{i=1}^{\frac{n}{2}} d_{i k} e^{\lambda_{i} s}+a_{k}, \quad k=3,5, \ldots, n-1$,

(ii) If the curve is unit speed $W$ - curve in $I E^{n}(\mathrm{n}=2 \mathrm{r}+1)$, then the curvature functions of the curve are

$$
\begin{aligned}
& m_{0}(s)=\sum_{i=1}^{\frac{n-1}{2}} c_{i} e^{-\lambda_{i} s}+\sum_{i=1}^{\frac{n-1}{2}} d_{i} e^{\lambda_{i} s}+a s+b, \\
& m_{1}(s)=\frac{1}{\kappa_{1}}\left(\sum_{i=1}^{\frac{n-1}{2}}-c_{i} \lambda_{i} e^{-\lambda_{i} s}+\sum_{i=1}^{\frac{n-1}{2}} d_{i} \lambda_{i} e^{\lambda_{i} s}+a-1\right), \\
& m_{j}(s)=\sum_{i=1}^{\frac{n-1}{2}} c_{i j} e^{-\lambda_{i} s}+\sum_{i=1}^{\frac{n-1}{2}} d_{i j} e^{\lambda_{i} s}+a_{j} s+b_{j}, \quad j=2,4, \ldots, n-1 \\
& m_{k}(s)=\sum_{i=1}^{\frac{n-1}{2}} c_{i k} e^{-\lambda_{i} s}+\sum_{i=1}^{\frac{n-1}{2}} d_{i k} e^{\lambda_{i} s}+a_{k}, \quad k=3,5, \ldots, n-1,
\end{aligned}
$$

where $a, a_{j}, a_{k}, b, b_{j}, c_{i}, c_{i j}, c_{i k}, d_{i}, d_{i j}, d_{i k}$ and $\lambda_{i}$ are real constants.

\section{Curves of Constant-ratio}

Definition 1: Let any curve $x: I \subset I R \rightarrow I E^{n}$ be unit speed in $I E^{n}$. The sum of the tangential and normal part of the curve gives its position vector as:

$x=x^{T}+x^{N}$.

In case of $\left\|x^{T}\right\|:\left\|x^{N}\right\|$ or $\left\|x^{T}\right\|:\|x\|$ is constant on all points of the curve, then $\mathrm{x}$ is called as a constant ratio curve (Chen, 2001).

Let $\rho=\|x(s)\|$ is the distance function and $\mathrm{T}$ is the tangent vector field, then the gradient is defined as $\operatorname{grad} \rho=\frac{d \rho}{d s} T(s)=\frac{\langle x(s), T(s)\rangle}{\|x(s)\|} T(s)$

Theorem 3: (Chen, 2003) Let any curve $x: I \subset I R \rightarrow I E^{n}$ be unit speed in $I E^{n}$. Then $\|$ grad $\rho \|$ is constant if and only if there exist three possible cases: 
(i) The curve is in the sphere of $I E^{n}$ where the center is the origin.

(ii) The curve is a line which passes the origin

(iii) The curve is given by $x(s)=\operatorname{cs} \alpha(s), c \in(0,1)$. Here, $\alpha=\alpha(t), t=\frac{\sqrt{1-c^{2}}}{c} \ln s$ is a unit speed curve and it lies on a sphere whose center is origin.

Corollary 2: (Chen, 2003) Let any curve $x: I \subset I R \rightarrow I E^{n}$ be unit speed in $I E^{n}$. Then

(i) $\|\operatorname{grad} \rho\|=0$ if and only if the curve is in a sphere of $I E^{n}$ whose center is the origin.

(ii) $\|\operatorname{grad} \rho\|=1$ if and only if the curve is a line which passes the origin.

(iii) $\|\operatorname{grad} \rho\|=c$ if and only if, for $c \in(0,1), c s=\|x(s)\|=\rho$.

(iv) In case of $\|\operatorname{grad} \rho\|=c, c \in(0,1)$ and two-dimensional Euclidean space, the curvature is given by

$\kappa^{2}=\frac{1-c^{2}}{c^{2}\left(s^{2}+a\right)}$

where a is a real constant.

The following result characterizes constant ratio curves in $I E^{n}$.

Proposition 1: Let any curve $x: I \subset I R \rightarrow I E^{n}$ be unit speed in $I E^{n}$. In case of $\mathrm{x}$ is a constant ratio, then it has the parametrization

$$
\begin{aligned}
x(s)= & \left(c^{2} s+b c\right) T(s)+\left(1-c^{2}\right)\left(\frac{-1}{\kappa_{1}(s)}\right) N_{1}(s) \\
& +\left(\frac{-\kappa_{1}^{2}(s)\left(c^{2} s+c b\right)}{\kappa_{2}(s)}\left(\frac{-1}{\kappa_{1}(s)}\right)+\frac{1-c^{2}}{\kappa_{2}(s)}\left(\frac{-1}{\kappa_{1}(s)}\right)^{\prime}\right) N_{2}(s) \\
& +\sum_{i=3}^{n-1}\left(\sum_{k=0}^{i-1} m_{i, k}(s) \frac{\partial^{k}}{\partial s^{k}}\left(\frac{-1}{\kappa_{1}(s)}\right)\right) N_{i}(s) .
\end{aligned}
$$

Here, $\mathrm{m}_{\mathrm{i}, \mathrm{k}}$ are inductively defined by the system 16 . where $m_{1,0}(s)=1-c^{2}$, $m_{2,0}(s)=\frac{-\kappa_{1}^{2}(s)\left(c^{2} s+c b\right)}{\kappa_{2}(s)}$, and $m_{2,1}(s)=\frac{1-c^{2}}{\kappa_{2}(s)}$.

Proof. Let any curve $x: I \subset I R \rightarrow I E^{n}$ be unit speed and constant ratio in $\mathrm{IE}^{\mathrm{n}}$. Then $\rho=\|x(s)\|=c s$. By the use of Equation 29., we obtain

$$
\|\operatorname{grad} \rho\|=\frac{\left\langle x(s), x^{\prime}(s)\right\rangle}{\|x(s)\|}=c .
$$


So, from the Equation 2. and 32., we yield $m_{0}=c^{2} s+c b$. Then, if we substitute it into Eq. 9, we have

$$
\begin{aligned}
& m_{1}(s)=\left(1-c^{2}\right)\left(\frac{-1}{\kappa_{1}(s)}\right) \\
& m_{2}(s)=\frac{-\kappa_{1}^{2}(s)\left(c^{2} s+c b\right)}{\kappa_{2}(s)}\left(\frac{-1}{\kappa_{1}(s)}\right)+\frac{1-c^{2}}{\kappa_{2}(s)}\left(\frac{-1}{\kappa_{1}(s)}\right)^{\prime} .
\end{aligned}
$$

By using the functions $m_{1,0}, m_{2,0}$, and $m_{2,1}$, we rewrite these equations as

$$
m_{1}(s)=m_{1,0}\left(\frac{-1}{\kappa_{1}(s)}\right) \quad \text { and } m_{2}(s)=m_{2,0}\left(\frac{-1}{\kappa_{1}(s)}\right)+m_{2,1}\left(\frac{-1}{\kappa_{1}(s)}\right)^{\prime}
$$

By induction method for equation system,

$$
m_{i}(s)=\sum_{k=0}^{i-1} m_{i, k}(s) \frac{\partial^{k}}{\partial s^{k}}\left(\frac{-1}{\kappa_{1}(s)}\right)
$$

for $i \in\{3,4, \ldots, n-1$,$\} . Here$

$$
\left\{\begin{array}{l}
m_{1,0}=1-c^{2} \\
m_{2,0}=\frac{-\kappa_{1}^{2}(s)\left(c^{2} s+c b\right)}{\kappa_{2}(s)}, \quad m_{2,1}=\frac{1-c^{2}}{\kappa_{2}(s)}
\end{array}\right.
$$

and for $i \in\{3,4, \ldots, n-1\}, m_{i, k}$ satisfy the Equation system 16 . As a result of these, substituting the values into $\mathrm{Eq} 2$., we get the result.

\section{T - Constant Curves}

Definition 2: Let any curve $x: I \subset I R \rightarrow I E^{n}$ be unit speed in $I E^{n}$. In case of $\left\|x^{T}\right\|$ is constant or zero on all points of the curve, then $\mathrm{x}$ is called as aT - constant curve (Gürpınar et al., 2015). Besides that, if $\left\|x^{T}\right\|=0$, then $\mathrm{x}$ is called $T$-constant curve of the first kind, if not second kind.

As a result of Eq. 9., we have the following theorem.

Theorem 4: Let any curve $x: I \subset I R \rightarrow I E^{n}$ be unit speed in $I E^{n}$ with the parametrization 2 . In case of $\mathrm{x}$ is a $T$-constant curve of the first kind, then it has the position vector as

$$
x(s)=\left(\frac{-1}{\kappa_{1}(s)}\right) N_{1}(s)+\frac{1}{\kappa_{2}(s)}\left(\frac{-1}{\kappa_{1}(s)}\right)^{\prime} N_{2}(s)+\sum_{i=3}^{n-1}\left(\sum_{k=0}^{i-1} m_{i, k}(s) \frac{\partial^{k}}{\partial s^{k}}\left(\frac{-1}{\kappa_{1}(s)}\right)\right) N_{i}(s) .
$$

Here, $m_{i, k}$ are inductively defined by the system 16 . where $m_{1,0}(s)=1, m_{2,0}(s)=0$, and $m_{2,1}(s)=\frac{1}{\kappa_{2}(s)}$. 
Proof. Let any curve $x: I \subset I R \rightarrow I E^{n}$ be $T$-constant first kind in $I E^{n}$. Then, from the Frenet equations 9., we obtain $m_{1}(s)=\frac{-1}{\kappa_{1}(s)}$ and $m_{2}(s)=\frac{1}{\kappa_{2}(s)}\left(\frac{-1}{\kappa_{1}(s)}\right)^{\prime}$. By using the functions $m_{1,0}, m_{2,0}$, and $m_{2,1}$, we rewrite these equations as

$m_{1}(s)=m_{1,0}\left(\frac{-1}{\kappa_{1}(s)}\right)$ and $m_{2}(s)=m_{2,0}\left(\frac{-1}{\kappa_{1}(s)}\right)+m_{2,1}\left(\frac{-1}{\kappa_{1}(s)}\right)^{\prime}$

By induction method for equation system,

$m_{i}(s)=\sum_{k=0}^{i-1} m_{i, k}(s) \frac{\partial^{k}}{\partial s^{k}}\left(\frac{-1}{\kappa_{1}(s)}\right)$

for $i \in\{3,4, \ldots, n-1$,$\} . Here$

$$
\left\{\begin{array}{l}
m_{1,0}=1-c^{2} \\
m_{2,0}=\frac{-\kappa_{1}^{2}(s)\left(c^{2} s+c b\right)}{\kappa_{2}(s)}, \quad m_{2,1}=\frac{1-c^{2}}{\kappa_{2}(s)}
\end{array}\right.
$$

and for $i \in\{3,4, \ldots, n-1\}, m_{i, k}$ satisfy the equation system 16. As a result of these, substituting the values into Eq. 2., we get the result.

Theorem 5: Let any curve $x: I \subset I R \rightarrow I E^{n}$ be unit speed in $I E^{n}$. In case of $\mathrm{x}$ is $T$-constant curve of second kind, then it has the position vector as

$$
x(s)=c T(s)+\left(\frac{-1}{\kappa_{1}(s)}\right) N_{1}(s)+\left(\frac{-\kappa_{1}^{2}(s) c}{\kappa_{2}(s)}\left(\frac{-1}{\kappa_{1}(s)}\right)+\frac{1}{\kappa_{2}(s)}\left(\frac{-1}{\kappa_{1}(s)}\right)^{\prime}\right) N_{2}(s)+\sum_{i=3}^{n-1}\left(\sum_{k=0}^{i-1} m_{i, k}(s) \frac{\partial^{k}}{\partial s^{k}}\left(\frac{-1}{\kappa_{1}(s)}\right)\right) N_{i}(s) .
$$

where $\mathrm{c}$ is a constant function.

Proof. Assume that $\mathrm{x}$ is $\mathrm{T}$-constant curve ofthe second kind $\left(m_{0}=c\right)$. Then, from by using Eq. 9., we obtain $m_{1}(s)=\frac{-1}{\kappa_{1}(s)}$ and $m_{2}(s)=\frac{-\kappa_{1}^{2}(s) c}{\kappa_{2}(s)}\left(\frac{-1}{\kappa_{1}(s)}\right)+\frac{1}{\kappa_{2}(s)}\left(\frac{-1}{\kappa_{1}(s)}\right)^{\prime}$. By using the functions $m_{1,0}, m_{2,0}$, and $m_{2,1}$, we rewrite these equations as

$m_{1}(s)=m_{1,0}\left(\frac{-1}{\kappa_{1}(s)}\right)$ and $m_{2}(s)=m_{2,0}\left(\frac{-1}{\kappa_{1}(s)}\right)+m_{2,1}\left(\frac{-1}{\kappa_{1}(s)}\right)^{\prime}$

By induction method for equation system,

$m_{i}(s)=\sum_{k=0}^{i-1} m_{i, k}(s) \frac{\partial^{k}}{\partial s^{k}}\left(\frac{-1}{\kappa_{1}(s)}\right)$

for $i \in\{3,4, \ldots, n-1$,$\} . Here$ 
$\left\{\begin{array}{l}m_{1,0}=1 \\ m_{2,0}=\frac{-\kappa_{1}^{2}(s) c}{\kappa_{2}(s)}, \quad m_{2,1}=\frac{1}{\kappa_{2}(s)}\end{array}\right.$

and for $i \in\{3,4, \ldots, n-1\}, m_{i, k}$ satisfy the equation system 16 . As a result of these, substituting the

values into Eq. 2., we get the result.

\section{N-Constant Curves}

Definition 3: Let any curve $x: I \subset I R \rightarrow I E^{n}$ be unit speed in $I E^{n}$. In case of $\left\|x^{N}\right\|$ is constant or zero on all points of the curve, then $\mathrm{x}$ is called as $N$-constant curve (Chen, 2002). In addition that, if $\left\|x^{N}\right\|=0$, then $\mathrm{x}$ is called a $N$-constant curve of the first kind, if not second kind (Gürpınar et al., 2015).

Thus, for the curve of $N$ - constant

$\left\|x^{N}(s)\right\|^{2}=\sum_{i=1}^{n-1} m_{i}^{2}(s)$

becomes a constant function.

As a result of Eq. 2., 9. and 44., we have the following:

Lemma 1: Let any curve $x: I \subset I R \rightarrow I E^{n}$ be unit speed in $I E^{n}$. The necessary and sufficient condition for $\mathrm{x}$ to become $N$-constant is

$$
\begin{aligned}
& m_{0}^{\prime}(s)=1+\kappa_{1}(s) m_{1}(s) \\
& m_{1}^{\prime}(s)=\kappa_{2}(s) m_{2}(s)-\kappa_{1}(s) m_{0}(s), \\
& m_{i}^{\prime}(s)=\kappa_{i+1}(s) m_{i+1}(s)-\kappa_{i}(s) m_{i-1}(s) \quad \text { with } i \in\{2,3, \ldots, n-2\} \\
& m_{n-1}^{\prime}(s)=-\kappa_{n-1}(s) m_{n-2}(s) \\
& 0=\sum_{i=1}^{n-1} m_{i}^{2}(s)
\end{aligned}
$$

hold, where $m_{i}(s), 1 \leq i \leq n-1$ are differentiable functions.

Theorem 6: Let any curve $x: I \subset I R \rightarrow I E^{n}$ be unit speed in $I E^{n}$. The necessary and sufficient condition for the curve to become $N$-constant first kind is that $\mathrm{x}$ is congruent to a line which passes the origin.

Proof. Assume that $\mathrm{x}$ is $N$-constant in $I E^{n}$, then the equation system 45 . holds. Further, $\mathrm{x}$ is a $N-$ constant curve of the first kind then from Eq. 45., $m_{i}=0$ for $i \in\{1,2, \ldots, n-1\}$. Namely, the parametrization is $x(s)=(s+b) T(s)$. Consequently, $\mathrm{x}$ is congruent to a line.

Theorem 7: Let a unit speed curve $x: I \subset I R \rightarrow I E^{n}$ has nonzero curvatures in $I E^{n}$. In case of $\mathrm{x}$ is a $N$-constant curve of the second kind, then the following cases are validated: 
(i) $\mathrm{x}$ is $T$-constant curve of first kind,

(ii) $\mathrm{x}$ is congruent to a rectifying curve in $I E^{n}$.

Proof. Let $\mathrm{x}$ is a $N$-constant curve of the second kind, then the equation system 45 . holds. By multiplying every $k$-th equations by $-m_{k-1}(1 \leq k \leq n)$ in Eq. 45., and combining them with the last equation in Eq. 45., we get $\kappa_{1}(s) m_{1}(s) m_{0}(s)=0$.

Since all curvatures are nonzero, there are two possible cases; $m_{0}=0, m_{1}=0$. If $m_{0}=0$, then $\mathrm{x}$ is of a $T$-constant first kind. Finally, if $m_{1}=0$, then $\mathrm{x}$ is congruent to a rectifying curve in $I E^{n}$.

\section{CONCLUSION}

$T$-constant, $N$-constant and constant ratio curves are first defined by B.Y. Chen. In this study, with regards to these definitions, we focus on these types of curves with Frenet frame in $n-$ dimensional Euclidean space and we present some results about $T$-constant, $N$-constant and constant ratio curves.

\section{REFERENCES}

Büyükkütük S, Öztürk G, 2015. Constant ratio curves according to Bishop frame in Euclidean 3-space $I E^{3}$. General Mathematics Notes, 28: 81-91.

Büyükkütük S, Kişi İ, Öztürk G, 2017. A characterization of curves according to paralel transport frame in Euclidean $n$-space $I E^{n}$. New Trends in Mathematical Sciences, 5: 61-68.

Büyükkütük S, Kişi İ, Mishra V. N, Öztürk G. 2016. Some characterizations of curves in Galilean 3 space $G_{3}$. Facta Universitatis-Series Mathematics and Informatics, 31: 503-512..

Chen BY, 2001. Constant ratio Hypersurfaces. Soochow Journal of Mathematics, 28: 353-362.

Chen BY, 2003. When does the position vector of a space curve always lies in its rectifying plane? The American Mathematical Monthly, 110: 147-152.

Chen BY, 2002. Geometry of warped products as Riemannian submanifolds and related problems. Soochow Journal of Mathematics, 28: 125-156.

Chen BY, 2003. More on convolution of Riemannian manifolds. Beitrage Zur Algebra Und Geometrie, 44: 9-27.

Chen BY, Dillen F, 2005. Rectifying curves as centrodes and extremal curves. Bulletin of theInsituteMathematics. Acedemia Sinica, 33: 77-90.

Cambie S, Geomans W, Bussche IVD, 2016. Rectifying curves in the $\mathrm{n}$-dimensional Euclidean space.Turkish Journalof Mathematics, 40: 210-223.

Gluck H, 1966. Higher curvatures of curvesin Euclidean space. The American Mathematical Monthly,73: 699-704.

Gray A, 1993. Modern differential geometry of curves and surfaces. CRS Press, Inc.

Gürpınar S, Arslan K, Öztürk G, 2015. A characterization of constant ratio curves in Euclidean 3 - space $I E^{3}$. Acta Universtatis Apulensis, 44: 39-51.

İlarslan K, Nesovic E, 2008. Some characterizations of rectifying curves in the Euclidean space $I E^{4}$. Turkish Journal of Mathematics, 32: 21-30. 
Kişi İ, Öztürk G, 2015. Constant ratio curves according to Bishop frame in Minkowski 3-space $I E_{1}^{3}$. Facta Universtatis, Series: Mathematics and Informatics, 30: 527-538.

Kişi İ, Büyükkütük S, Öztürk G, 2018. Constant ratio timelike curves in pseudo-Galilean 3 -space $G_{3}^{1}$. Creat. Math. Inform., 27: 57-62.

Kişi İ, Büyükkütük S, Öztürk G, Zor A, 2017. A new characterization of curves on dual unit sphere. Journal of Abstract and Computational Mathematics, 2: 71-76.

Klein F, Lie S, 1871. Uber diejenigen ebenenen kurven welche durch ein geschlossenes system von einfach unendich vielen vartauschbaren Transformationen in sich ubergeben. Mathematische Annalen, 4: 5084.

Öztürk G, Arslan K, Kişi İ, 2018. Constant ratio curves in Minkowski 3 - space $I E_{1}^{3}$. Matematicki Bilten, 42: 49-60.

Öztürk G, Büyükkütük S, Kişi İ, 2017. A characterization of curves in Galilean 4-space $G_{4}$. Bulletin of the Irannian Mathematical Society, 43: 771-780.

Öztürk G, Kişi İ, Büyükkütük S, 2017. Constant ratio quaternionic curves in Euclidean spaces. Applied Clifford Algebras, 27: 1659-1673. 